\title{
Sensation Seeking Scale
}

National Cancer Institute

\section{Source}

National Cancer Institute. Sensation Seeking Scale. NCI Thesaurus. Code C121284.

A rating scale included in the Behavior Assessment System for Children that measures

the tendency of the subject to take risks and seek excitement. 